\title{
Estado nutricional, actividad física y consumo de comida chatarra asociados a colelitiasis en huánuco. Estudio de casos y controles
}

\author{
Edgar Agurto-Jara ${ }^{1}$, Claudia Espinoza-Cardich ${ }^{1}$, Bernardo Dámaso-Mata ${ }^{1,2}$, \\ Kovy Arteaga-Livias ${ }^{1,2}$ y Vicky Panduro-Correa ${ }^{1,3}$
}

\section{Nutritional status, physical activity and consumption of junk food were associating to colelitiasis in huánuco. A case-control study}

\begin{abstract}
Aim: To determine the association between junk food consumption, physical activity and nutritional status in patients with cholelithiasis on ultrasound (cases), compared with apparently healthy controls without cholelithiasis on ultrasound, in an outpatient EsSalud II Hospital, Huánuco Hospital, 2017. Materials and Method: Case-control study. 107 cases and 107 controls were included. The consumption of junk food was assessed with the survey validated by RSEQ (Québec Student Sports Network), the frequency of consumption (days per week); Physical activity through the IPAQ questionnaire (International Physical Activity Questionnaire) and nutritional status through the Body Mass Index. The chi-square, Mann Whitney $U$ and Odds Ratio $(\mathrm{p}<0.05)$ were used in the statistical analysis. Results: Gender, age and origin were not significant. None or low consumption of "junk" food were associated with cholecystitis (OR 0.097; $95 \%$ CI $0.052-0.182)$. Low physical activity $(<600 \mathrm{MET})$ did not promote the diagnosis of cholecystitis (OR 0.119; 95\% CI 0.064-0.22) and the nutritional status (normal weight), significantly, did not promote cholecystitis (OR 0.09; 95\% CI 0.05-0.167). Being male was associated with the non-development of cholecystitis (OR 0.48; 95\% CI 0.281-0.838). Conclusion: Low or no consumption of "junk" food, low physical activity (<600 MET), nutritional status (normal) and gender (male) were associated for the nondevelopment of cholelithiasis diagnosed by ultrasound.
\end{abstract}

Key words: cholelithiasis; exercise; fast food; nutritional status; body mass index.

\section{Resumen}

Objetivos: Determinar la asociación entre el consumo de comida chatarra, actividad física y estado nutricional en pacientes con colelitiasis a la ecografía (casos), comparados con controles aparentemente sanos sin colelitiasis a la ecografía, en consulta externa del Hospital EsSalud II Huánuco. Materiales y Método: Estudio de casos y controles. Se incluyeron 107 casos y 107 controles. El consumo de comida "chatarra" se valoró con la encuesta validada por RSEQ (Red deportiva estudiantil de Québec), la frecuencia del consumo (días por semana); la actividad física por el cuestionario IPAQ (Cuestionario Internacional de Actividad Física) y el estado nutricional mediante el índice de masa corporal. En el análisis estadístico se empleó el $\chi^{2}$, U de Mann Whitney y Odds Ratio $(\mathrm{p}<0,05)$. Resultados: La edad y la procedencia no fueron significativos. El consumo de comida "chatarra" estuvo asociado a la colecistitis (OR 10,30; IC 95\% 5,48-19,37). La actividad física moderada-vigorosa ( $\geq 600 \mathrm{MET}$ ) promovió el diagnóstico de colecistitis (OR 8,38; IC 95\% 4,54-15,48) y el estado nutricional (sobrepeso o mayor), en forma significativa, promovía la colecistitis (OR 11,38; IC 95\% 6,01-21,55). Ser femenino estuvo asociado al desarrollo de colecistitis (OR 2,06; IC 95\% 1,19-3,55). Conclusión: El consumo de comida "chatarra", la actividad física moderada-vigorosa ( $\geq 600 \mathrm{MET}$ ), el estado nutricional (sobrepeso o mayor) y el género (femenino) fueron asociados al desarrollo de colelitiasis diagnosticados mediante ecografía.

Palabras clave: colelitiasis; ejercicio; comida rápida; estado nutricional; índice de masa corporal.
'Facultad de Medicina. Universidad Nacional Hermilio Valdizán. Huánuco, Perú. ${ }^{2}$ Hospital II EsSalud. Huánuco, Perú.

${ }^{3}$ Hospital Regional Hermilio Valdizán. Huánuco, Perú.

Contribuciones de los autores: EAJ, CEC y VPC participaron en la concepción del estudio, EAJ y CEC, realizaron la recogida de datos y realizaron la confección del manuscrito, KAL, BDM y VPC, revisaron el análisis estadístico y realizaron la revisión crítica del manuscrito. Todos los autores aprobaron la versión final. Fuentes de financiamiento: El proyecto fue financiado por los autores.

Recibido el 2 de mayo de 2019 y aceptado para publicación el 5 de diciembre de 2019.

Correspondencia a: Dra. Vicky Panduro-Correa vpanduro@unheval.edu.pe 


\section{Introducción}

La colelitiasis es una enfermedad caracterizada por la presencia de cálculos dentro de la vesícula biliar (VB). La etiología de los cálculos es multifactorial, considerándose con factores predisponentes la obesidad, embarazo, edad avanzada, factores dietéticos, enfermedad de Crohn, resección ileal terminal, operación gástrica, esferocitosis hereditaria, enfermedad de células falciformes y talasemia ${ }^{1}$.

Los cálculos de colesterol, que son los más frecuentes, están asociados a un incremento del colesterol en la bilis, lo que aumenta su densidad dentro de la vesícula, que junto a niveles elevados de proteínas proinflamatorias como interleucina-6 (IL6), IL-10. IL-12 e IL-13, hipersecreción de gel de mucina, parecen condicionar el riesgo de presentar colelitiasis. Existen complejas vías metabólicas que podrían condicionar el desarrollo de cálculos biliares asociadas a enfermedades metabólicas ${ }^{2}$.

A nivel mundial, la colecistitis afecta a millones de personas en todo el mundo, sobre todo, en las sociedades occidentales, donde se diagnostica entre un $10 \%$ y un $30 \%$ de sus habitantes y cada año hay un millón de casos nuevos. La carga de la enfermedad, en Estados Unidos representa 700.000 colecistectomias anuales ${ }^{3}$.

En América Latina se informa que entre el 5 y el $15 \%$ de los habitantes presentan litiasis vesicular, y existen poblaciones y etnias con mayor prevalencia, como la caucásica, la hispánica o los nativos americanos. Países como Estados Unidos, Chile, Bolivia se encuentran entre los de mayor número de personas afectadas por litiasis vesicular ${ }^{4}$.

En Perú, en el año 2015, se realizó un estudio en el Hospital Hipólito Unanue (Lima) donde se demostró las características más prevalentes de su realidad; el grupo masculino era el más afectado (58\%); el grupo etario más afectado fue el de 36 años a 45 años (51\%); en relación a las comorbilidades la obesidad fue la de mayor frecuencia $(17 \%)$ y en cuanto al consumo de dieta rica en fibra, el $78 \%$ no lo consumía ${ }^{5}$.

Como se detalla estadísticamente esta patología multifactorial conlleva importantes implicaciones médicas, sociales y económicas, por su elevada frecuencia y su tratamiento quirúrgico es una de las cirugías abdominales que más frecuentemente se realizan a nivel mundial ${ }^{6}$.

Por lo anterior, se planteó como objetivo el evaluar la asociación del estado nutricional, la actividad física y el consumo de comida chatarra a la colelitiasis, comparados con controles aparentemente sanos sin colelitiasis diagnosticados por ecografía en los pacientes atendidos en consulta externa del Hospital EsSalud II Huánuco, Perú.

\section{Materiales y Método}

\section{Contexto}

El Hospital II de la Red Asistencial de Huánuco EsSalud, forma parte del sistema del Seguro Social de Salud (EsSalud), ubicado en la región centrooriente del Perú (sierra y selva), brindando servicios integrales de salud a su población asegurada; trabajadores, los derechohabientes, pensionistas y otros. La población coberturada a junio de 2019 , fue de cerca de 172.383 personas, constituida principalmente por población trabajadora (con nivel educativo predominante secundario a superior).

\section{Tipo de estudio}

Estudio de casos y controles.

\section{Población, muestra y muestreo}

La población correspondió a los pacientes que acudieron a la consulta externa del Hospital II Es Red Asistencial Huánuco EsSalud recolectados entre julio y noviembre de 2017.

Respecto al tamaño de la muestra, se buscó antecedentes de estudios basados en las variables de interés y que hayan sido elaboradas con un diseño similar al nuestro, por lo cual, se empleó la investigación titulada: "A nested case-control study on dietary fat consumption and the risk for gallstone disease" , con una proporción para los expuestos de $44 \%$ y de los no expuestos de $22 \%$, con un nivel de confianza del $95 \%$, una potencia del $80 \%$, una relación caso: control de 1:1, obteniéndose un tamaño de 107 casos y 107 controles. El muestreo fue no probabilístico por conveniencia.

\section{Criterios de elegibilidad}

Se consideró caso a todo paciente con diagnóstico ecográfico de colelitiasis (independientemente si fueron o no operados por este motivo) atendidos en el servicio de consulta externa del Hospital EsSalud II-Huánuco en el año 2017 (período de julio a noviembre). Para su selección se fue tomando cada caso hasta conseguir el número requerido. Para los controles se consideraron a los pacientes que no tuvieron colelitiasis en el estudio ecográfico. Los controles se seleccionaron de la misma manera que los casos. Como criterios de exclusión se consideró el padecimiento de hipertensión arterial, diabetes mellitus tipo 2 , enfermedad arterial coronaria y 
cerebrovascular, dislipidemia, síndrome metabólico, apnea del sueño, hígado graso no alcohólico, enfermedad por reflujo gastroesofágico, Acantosis nigricans, estrías, osteoartritis degenerativas, síndrome de ovario poliquístico, intolerancia a la glucosa y pacientes que no aceptaron participar en la investigación.

\section{Variables}

La variable dependiente fue la presencia o ausencia de colelitiasis a la ecografía (casos y controles respectivamente).

Definición de caso: se definieron como aquellos sujetos cuyas colelitiasis fueron diagnosticadas por la presencia de una o más estructuras móviles ecogénicas dentro de la vesícula biliar en el estudio de ultrasonido.

Definición de control: fueron seleccionados por conveniencia sin diagnóstico aparente de colelitiasis, que en el ultrasonido no se encontraba ninguna estructura móvil dentro de la vesícula biliar.

Las variables independientes fueron el estado nutricional (se empleó el IMC [peso entre talla al cuadrado, $\mathrm{kg} / \mathrm{m}^{2}$ ], la actividad física (medido a través del Cuestionario Internacional de Actividad Física [IPAQ]: baja [<600 MET], moderada [600-1.500 MET] y vigorosa [ $>1.500 \mathrm{MET}])$ y el consumo de comida chatarra (cuestionario de comida chatarra, bebidas endulzadas y energéticas: Consumo y opiniones de los jóvenes de Quebec. Validado por RSEQ). Un MET se define como el costo energético de sentarse en silencio y es equivalente al consumo calórico de $1 \mathrm{kcal} / \mathrm{kg} / \mathrm{h}$.

La recolección de los datos se realizó mediante cuestionarios aplicados en la entrevista personal a los pacientes atendidos en consulta externa del Hospital II EsSalud Huánuco en el 2017, previamente validada por juicio de expertos.

\section{Análisis estadístico}

Se realizó un análisis descriptivo con la medición de frecuencias y de dispersión. La relación de las variables cualitativas con la colelitiasis se estableció mediante la prueba de $\chi^{2}$. En el análisis final no hubo cálculos entre las variables cuantitativas, por lo que no se utilizaron otras pruebas estadísticas. La medida de fuerza de asociación se calculó con la prueba de Odds Ratio (OR con IC 95\%). Se emplearon los programas estadísticos STATA 12.0 y SPSS 15.0.

\section{Aspectos éticos}

Para la realización de las entrevistas se solicitó su autorización para participar, se aplicó consen- timiento informado, explicando los alcances de la investigación y ejecutando posteriormente el cuestionario. El proyecto fue aprobado por el Comité de Ética e Investigación del Hospital II EsSalud Red Asistencial de Huánuco.

\section{Resultados}

Se obtuvo datos de 214 participantes, 107 casos y 107 controles. Al analizar las características epidemiológicas presentadas en la Tabla 1, resalta que más de la mitad de encuestados tenían un grado alto de preparación académica, siendo esta el $63 \%$ de la población estudiada, además, no existe una gran diferencia en cuanto a la cantidad de participantes dependiendo del género (46\% masculino y $56 \%$ femenino), mencionar también que casi no se contó con la participación de población adulta mayor ( $\geq 65$ años) para que de esta manera se tenga certeza de que hubo una total comprensión por los participantes, siendo el promedio de edad de los participantes de 42,7 $\pm 10,60$.

Las características clínicas son presentadas en la Tabla 2, se resalta que había un grupo importante de pacientes con sobrepeso, pero que presentaban cierto grado de actividad física aceptable.

Tabla 1. Características epidemiológicas de los pacientes atendidos en consulta externa del Hospital II EsSalud Red Asistencial Huánuco 2017 (n= 214)

\begin{tabular}{|lrc|}
\hline Característica & Frecuencia & Porcentaje \\
\hline Género & & \\
Masculino & 99 & $46 \%$ \\
Femenino & 115 & $54 \%$ \\
\hline Nivel educativo & & \\
Analfabeto & 2 & $1 \%$ \\
Primaria incompleta & 6 & $3 \%$ \\
Primaria completa & 3 & $1 \%$ \\
Secundaria incompleta & 20 & $9 \%$ \\
Secundaria completa & 10 & $5 \%$ \\
Técnica & 7 & $3 \%$ \\
Universitaria incompleta & 31 & $15 \%$ \\
Universitaria completa & 135 & $63 \%$ \\
\hline Edad & & \\
X \pm DS & & $42,7 \pm 10,60$ \\
\hline
\end{tabular}

X: Media aritmética. DS: Desviación estándar. 
Tabla 2. Características clínicas de los pacientes atendidos en consulta externa del Hospital II EsSalud Red Asistencial Huánuco 2017 (n= 214)

\begin{tabular}{|c|c|c|}
\hline Característica & Frecuencia & Porcentaje \\
\hline \multicolumn{3}{|l|}{ Diagnóstico ecográfico de colelitiasis } \\
\hline Sí & 107 & $50 \%$ \\
\hline No & 107 & $50 \%$ \\
\hline \multicolumn{3}{|l|}{ Estado nutricional } \\
\hline \multicolumn{3}{|l|}{ IMC } \\
\hline $\mathrm{X} \pm \mathrm{DS}$ & \multicolumn{2}{|c|}{$25,81 \pm 3,5$} \\
\hline Bajo peso & 0 & $0 \%$ \\
\hline Peso normal & 104 & $49 \%$ \\
\hline Sobrepeso & 89 & $41 \%$ \\
\hline Obesidad grado I & 16 & $8 \%$ \\
\hline Obesidad grado II & 5 & $2 \%$ \\
\hline Obesidad grado III & 0 & $0 \%$ \\
\hline \multicolumn{3}{|l|}{ Número de familiares que han tenido colelitiasis } \\
\hline Cero $(0)$ & 106 & $50 \%$ \\
\hline Uno (1) & 76 & $35 \%$ \\
\hline Dos (2) & 28 & $12 \%$ \\
\hline Tres (3) & 3 & $2 \%$ \\
\hline Cuatro (4) & 1 & $1 \%$ \\
\hline \multicolumn{3}{|l|}{ Consumo de comida chatarra } \\
\hline \multicolumn{3}{|l|}{ Comida frita } \\
\hline Nunca & 22 & $10 \%$ \\
\hline Rara vez & 72 & $34 \%$ \\
\hline 2 veces por semana & 19 & $9 \%$ \\
\hline 3 o 4 veces por semana & 60 & $28 \%$ \\
\hline Todos o casi todos los días & 41 & $19 \%$ \\
\hline \multicolumn{3}{|l|}{ Comida chatarra } \\
\hline Nunca & 40 & $19 \%$ \\
\hline Rara vez & 62 & $29 \%$ \\
\hline 2 veces por semana & 7 & $3 \%$ \\
\hline 3 o 4 veces por semana & 69 & $32 \%$ \\
\hline Todos o casi todos los días & 36 & $17 \%$ \\
\hline \multicolumn{3}{|l|}{ Actividad física } \\
\hline Actividad física baja $(<600$ MET) & 104 & $49 \%$ \\
\hline Actividad física moderada (600-1.500 MET) & 42 & $20 \%$ \\
\hline Actividad física vigorosa (> $1.500 \mathrm{MET})$ & 68 & $31 \%$ \\
\hline
\end{tabular}

X: Media aritmética. DS: Desviación estándar.
En cuanto a los participantes con antecedentes de familiares de colelitiasis no mostró una gran diferencia, ya que aproximadamente el 40\% de controles sí tenían estos antecedentes, pero de un solo familiar, sin embargo, algunos casos tenían antecedentes hasta 4 familiares con colelitiasis. El estado nutricional promedio, representado por el IMC, fue de 25,81 $\pm 3,5$, siendo los participantes con peso normal y sobrepeso los de mayor cantidad, representando el $49 \%$ y el $41 \%$ respectivamente. En cuanto al consumo de comida chatarra hubo una gran diferencia en cuanto a casos y controles, ya que los casos lo consumían en mayor frecuencia por semana, igualmente resaltar que la respuesta más común para controles fue consumir rara vez (la cual para los autores fue tomado como una vez por semana) y para los casos fue de 3 a 4 veces por semana. El nivel de actividad física de mayor frecuencia fue el bajo con $49 \%$, destacando que una buena parte de controles realizaban esta actividad física baja y que algunos pocos casos realizaban actividad física vigorosa.

En la Tabla 3 se demuestran los resultados del análisis bivariado realizado, demostrando que fueron estadísticamente significativas las variables género, actividad física, estado nutricional y el consumo de comida chatarra con relación a la colelitiasis diagnosticado por ecografía.

\section{Discusión}

La colelitiasis es una de las patologías más frecuentes que se diagnostican en los servicios de medicina y cirugía principalmente. En relación a la incidencia de colelitiasis encontramos que es un asunto de gran magnitud, por lo cual es significativo como problema médico-quirúrgico y socioeconómico en nuestros sistemas de salud actuales ${ }^{8}$.

El estado nutricional, representado por el IMC (índice de masa corporal) en nuestro estudio resultó con significación estadística; se evidenció que los pacientes que no tenían cálculos en la vesícula tenían peso normal a bajo peso, a diferencia de los que sí tenían colelitiasis (diagnosticados por ecografía abdominal) presentaban el rango de sobrepeso a obesidad; esto se apoya con lo encontrado por Aune, que concluyen que un aumento del IMC por encima del intervalo normal incrementa el riesgo de litiasis vesicular ${ }^{9}$, así también un metaanálisis de estudios de cohortes encontró que la dislipidemia y la obesidad son los factores determinantes para el desarrollo de litiasis biliar siendo estos modificables por lo que es posible disminuir la frecuencia de esta dolencia actuando sobre los mismos ${ }^{10}$, del mismo modo un 
ARTíCULO ORIGINAL

Tabla 3. Análisis bivariado de las características de los pacientes atendidos en consultorio externo en el Hospital II EsSalud Red Asistencial Huánuco $2017(\mathrm{n}=\mathbf{2 1 4})$

\begin{tabular}{|c|c|c|c|c|c|c|c|c|}
\hline \multirow[t]{2}{*}{ Características } & \multicolumn{4}{|c|}{ Colelitiasis (por ecografía) } & \multirow[t]{2}{*}{ p* } & \multirow[t]{2}{*}{ OR } & \multicolumn{2}{|c|}{ IC $95 \%$} \\
\hline & Sí & $\%$ & No & $\%$ & & & Inferior & Superior \\
\hline \multicolumn{9}{|l|}{ Género } \\
\hline Masculino & 40 & $40,40 \%$ & 59 & $59,60 \%$ & & & & \\
\hline \multicolumn{9}{|l|}{ Estado nutricional } \\
\hline Sobrepeso o mayor & 84 & $76,00 \%$ & 26 & $24,00 \%$ & 0,001 & 11,38 & 6,01 & 21,55 \\
\hline Bajo peso-peso normal & 23 & $22,10 \%$ & 81 & $77,90 \%$ & & & & \\
\hline \multicolumn{9}{|l|}{ Consumo de comida chatarra } \\
\hline \multicolumn{9}{|l|}{ Comida frita } \\
\hline 2 o más días por semana & 84 & $75,00 \%$ & 28 & $25,00 \%$ & 0,001 & 10,30 & 5,48 & 19,37 \\
\hline Nunca-Rara vez & 23 & $22,50 \%$ & 79 & $77,50 \%$ & & & & \\
\hline \multicolumn{9}{|l|}{ Actividad física } \\
\hline Actividad física moderada-vigorosa ( $\geq 600 \mathrm{MET})$ & 78 & $75,00 \%$ & 26 & $25,00 \%$ & 0,001 & 8,38 & 4,54 & 15,48 \\
\hline Actividad física baja (<600 MET) & 29 & $26,00 \%$ & 81 & $74,00 \%$ & & & & \\
\hline
\end{tabular}

* Test de $\chi^{2}$.

estudio europeo encuentra que existe una relación significativa entre el IMC incrementado y el riesgo de colelitiasis sintomática ${ }^{11}$. Contrariamente, Ordeñada, encontró en su investigación que la obesidad fue poco significativa para el desarrollo de litiasis vesicular, lo cual podría deberse a la metodología y tipo de estudio ${ }^{12}$, sin embargo, el estudio de Kharga y sus colaboradores concluyeron que no solo los pacientes con sobrepeso u obesos desarrollan colelitiasis sintomática, sino también las personas con un IMC normal, pero que el riesgo de colelitiasis sintomática aumenta con cada aumento en el $\mathrm{IMC}^{13}$. Adicionalmente, un estudio realizado en la ciudad de Huánuco, no encuentra asociación entre la presencia de síndrome metabólico y colelitiasis o colecistectomía ${ }^{14}$.

El consumo de comida chatarra en nuestra investigación resultó con significación estadística; se evidenció que los pacientes que no tenían cálculos en la vesícula tenían un consumo de comida chatarra menor de 2 días a la semana; a diferencia de los que sí tenían colelitiasis (diagnosticados por ecografía abdominal) donde consumían por lo menos 2 veces a la semana comida chatarra; esto se apoya con lo encontrado por los estudios de Compagnucci y Park quienes demostraron que el consumo de grasas saturadas y monoinsaturadas incrementaban el riesgo de cálculos en la vesícula ${ }^{7,15}$, adicionalmente a esto, Lander encontró que el consumo de proteínas vegetales disminuía el riesgo de padecer cálculos de la vesícula ${ }^{16}$. En Chile, del Pozo, en un estudio experimental en ratones concluye que la ingestión de una dieta alta en grasas, y en mayor predominio grasas saturadas, podría contribuir a la formación de cálculos de colesterol en la VB, principalmente, en un estadio temprano ${ }^{17}$. A diferencia de los estudios mencionados, encontramos que Johansson y sus colaboradores hallaron que el riesgo de cálculos biliares sintomáticos que requieren hospitalización o colecistectomía (aunque bajo) fue 3 veces mayor con VLCD (dieta muy baja en calorías) que LCD (dieta baja en calorías) durante el programa de pérdida de 
peso comercial de 1 año; a pesar de esta diferencia este factor no fue significativamente estadístico para su estudio ${ }^{18}$; también, Brindal y colaboradores, determinaron que la frecuencia de consumo (por mes) de comida rápida fue de 3,40 y que mayoría de las personas $(63,3 \%)$ reportaron comer comida rápida entre una vez al mes y una vez a la semana; esto tuvo un mayor riesgo de desarrollar colelitiasis, en contraste con nuestros resultados ${ }^{19}$ y agregando, finalmente, el estudio de Wiesław Pilis y colaboradores donde demostraron que una dieta vegetariana, incluso siendo hipercalórica, si es adecuadamente equilibrada puede conducir a evitar enfermedades relacionadas a la obesidad ${ }^{20}$.

La actividad física finalmente en nuestra investigación resultó con significación estadística; se evidenció que los pacientes que tenían cálculos en la vesícula tenían una actividad física moderada a vigorosa según la encuesta utilizada (IPAQ-Cuestionario Internacional de Actividad Física), a diferencia de aquellos que no tenían colelitiasis (diagnosticados por ecografía abdominal) quienes presentaban una baja actividad física. Esto es contrario con lo hallado por Zhang en un estudio de metaanálisis donde concluye que existe una relación inversa entre la actividad física y el riesgo de colecistectomía por colelitiasis y la actividad física se asoció con un menor riesgo de colelitiasis sintomática ${ }^{3}$, igualmente en el estudio realizado por Henao donde se demostró que la actividad física recreacional protege contra los cálculos biliares asintomáticos ${ }^{21}$, y apoyan a lo mencionado por Aune y cols, con su estudio donde el ejercicio físico fue un factor protector contra el desarrollo de litiasis vesicular ${ }^{22}$. Semejante a nuestros hallazgos encontramos que Martin, en su estudio, reporta que el ejercicio físico intenso podría ser un factor de riesgo para desarrollar litiasis vesicular $^{23}$, como lo mostrado por nosotros donde la actividad vigorosa-moderada fue más frecuente en los pacientes con colelitiasis; del mismo modo Shephard encontró que la actividad física no tuvo un gran impacto en el desarrollo de cálculos en la vesícula en su estudio ${ }^{24} \mathrm{y}$, finalmente, Keum encontró en su estudio que el beneficio óptimo se observó a través del ejercicio aeróbico de cualquier intensidad en el equivalente del gasto de energía de aproximadamente $10 \mathrm{~h} / \mathrm{semana}$ de caminata al ritmo promedio, interpretado como actividad física baja según la encuesta IPAQ (Cuestionario Internacional de Actividad Física) ${ }^{25}$.

Dentro de las limitaciones del presente estudio se tiene que no se dispone de una población específica a atender en consulta externa del Hospital EsSaludII Huánuco, lo que limita a establecer un mínimo teórico de muestra para la evaluación y no se dispone de estudios nacionales ni regionales profundos ni actualizados sobre la asociación de comida chatarra y colelitiasis.

En conclusión, el consumo de comida "chatarra", la actividad física moderada-vigorosa ( $\geq 600 \mathrm{MET})$, el estado nutricional (sobrepeso o mayor) y el género (femenino) fueron asociados al desarrollo de colelitiasis diagnosticados por ecografía.

\section{Agradecimientos}

Agradecemos a nuestra colega Macarena Carbajal Villanueva por su colaboración en la elaboración del estudio.

\section{Responsabilidades éticas}

Protección de personas y animales. Los autores declaran que para esta investigación no se han realizado experimentos en seres humanos ni en animales.

Confidencialidad de los datos. Los autores declaran que en este artículo no aparecen datos de pacientes.

Conflictos de interés: no hay.

\section{Bibliografía}

1. Schwartz SI, Brunicardi FC, Andersen DK. Principios de cirugía [de] Schwartz. México, McGraw-Hill Interamericana; 2011.

2. Di Ciaula A, Portincasa P. Recent advances in understanding and managing cholesterol gallstones. F1000Res. 2018;7:F1000 Faculty Rev-1529.
Published 2018 Sep 24. DOI: 10.12688/ f1000research.15505.1

3. Zhang YP, Zhao YL, Sun YL, Zhu RT, Wang WJ, Li J. Physical Activity and the Risk of Gallstone Disease: A Systematic Review and Meta-analysis. J Clin Gastroenterol. 2017;51:857-68. DOI: 10.1097/MCG.0000000000000571

4. Gonzales Maticorena. Factores de riesgo asociados a pacientes con diagnóstico de colecistitis crónica calculosa en el Hospital Nacional Hipólito Unanue en el 2015. 2017 [citado el 21 de mayo de 2017]; Disponible en: http://cybertesis. urp.edu.pe/handle/urp/881.

5. Panduro Delgado AC. Causas de conversión de la colecistectomía laparascópica, Departamento de Cirugía, Hospital III Iquitos-EsSalud, octubre 2013 a octubre 2014. 2015 [citado el 21 
de mayo de 2017]; Disponible en: http:// repositorio.unapiquitos.edu.pe/handle/ UNAP/3750.

6. Roesch-Dietlen F, Pérez-Morales AG, Martínez-Fernández S, Díaz-Roesch F, Gómez-Delgado JA, Remes-Troche JM. Safety of laparoscopic subtotal cholecystectomy in acute cholecystitis. Experience in Southeast Mexico. Seguridad de la colecistectomía subtotal laparoscópica en colecistitis aguda. Experiencia en el sureste de México. Rev Gastroenterol Mex. 2019;84:461-6. DOI: 10.1016/j.rgmx.2018.11.012.

7. Compagnucci A, Perroud HA, Batallés SM, Villavicencio R, Brasca A, Berli $\mathrm{D}$, et al. A nested case-control study on dietary fat consumption and the risk for gallstone disease. J Hum Nutr Diet. 2016;29:338-44.

8. Lammert F, Gurusamy K, Ko CW, Miquel JF, Méndez-Sánchez N, Portincasa P, et al. Gallstones. Nat Rev Dis Primers. 2016;2:16024. Published 2016 Apr 28. DOI: $10.1038 /$ nrdp.2016.24.

9. Aune D, Norat T, Vatten LJ. Body mass index, abdominal fatness and the risk of gallbladder disease. Eur J Epidemiol. 2015;30:1009-19.

10. Shabanzadeh DM, Sørensen LT, Jørgensen T. Determinants for gallstone formation - a new data cohort study and a systematic review with meta-analysis. Scand J Gastroenterol. 2016;51:1239-48. DOI: 10.1080/00365521.2016.1182583.

11. McConnell TJ, Appleby PN, Key TJ. Vegetarian diet as a risk factor for symptomatic gallstone disease. Eur J Clin Nutr. 2017;71:731-5. DOI: 10.1038/ ejen.2016.252.

12. Ordeñana-Chevez ES. Colelitiasis: factores de riesgo y complicaciones en adultos de 30 a 60 años de edad. Estudio a realizar en el Hospital Universitario de Guayaquil periodo 2015. Universidad de Guayaquil. Facultad de Ciencias Médicas. Escuela de Medicina; 2016.

13. Kharga B. Obesity Not Necessary, Risk of Symptomatic Cholelithiasis Increases as a Function of BMI. J Clin Diagn Res [Internet]. 2016 [citado 26 de noviembre de 2017]; Disponible en: http://jcdr.net/ article_fulltext.asp?issn=0973-709x\&year $=2016 \&$ volume $=10 \&$ issue $=10 \&$ page $=$ PC 28\&issn=0973-709x\&id=8736.

14. Fierro-Barzola W, Ureta M, Niquén CJ. Litiasis Biliar o Colecistectomía Asociada a Síndrome Metabólico en Pacientes Del Hospital II - EsSalud, Huánuco. Rev Peru Investig Salud. 2018;2(1):50-5. Available from: http://revistas.unheval.edu.pe/index. php/repis/article/view/213.

15. Park Y, Kim D, Lee JS, Kim YN, Jeong YK, Lee KG, et al. Association between diet and gallstones of cholesterol and pigment among patients with cholecystectomy: a case-control study in Korea. J Health Popul Nutr. 2017;36:39. Published 2017 Nov 23. DOI: 10.1186/ s41043-017-0116-y.

16. Lander EM, Wertheim BC, Koch SM, Chen Z, Hsu C-H, Thomson CA. Vegetable protein intake is associated with lower gallbladder disease risk: Findings from the Women's Health Initiative prospective cohort. Prev Med. 2016;88:20-6.

17. Del Pozo R, Mardones L, Villagrán M, Muñoz K, Roa S, Rozas F, et al. Efecto de una dieta alta en grasas en el proceso de formación de cálculos biliares de colesterol. Rev Med Chile 2017;145:1099-105. DOI: 10.4067/s003498872017000901099 .
18. Johansson K, Sundström J, Marcus C, Hemmingsson E, Neovius M. Risk of symptomatic gallstones and cholecystectomy after a very-low-calorie diet or low-calorie diet in a commercial weight loss program: 1-year matched cohort study. Int J Obes. 2014;38:279-84.

19. Brindal E, Wilson C, Mohr P, Wittert G. Nutritional consequences of a fast food eating occasion are associated with choice of quick-service restaurant chain: Fast food consumption and choice of QSR chain. Nutr Diet. 2014;71:184-92.

20. Pilis W, Stec K, Zych M, Pilis A. Health benefits and risk associated with adopting a vegetarian diet. Rocz Państw Zakl Hig. 2014;65:9-14.

21. Henao-Morán S, Denova-Gutiérrez E, Morán S, Duque X, Gallegos-Carrillo K, Macías N, et al. Recreational physical activity is inversely associated with asymptomatic gallstones in adult Mexican women. Ann Hepatol Of J Mex Assoc Hepatol. 2014;13:810-8.

22. Aune D, Leitzmann M, Vatten LJ. Physical Activity and the Risk of Gallbladder Disease: A Systematic Review and Meta-Analysis of Cohort Studies. J Phys Act Health. 2016;13:78895.

23. Martin D. Physical Activity Benefits and Risks on the Gastrointestinal System: South Med J. 2011;104:831-7.

24. Shephard RJ. Physical Activity and the Biliary Tract in Health and Disease. Sports Med. 2015;45:1295-309.

25. Keum N, Bao Y, Smith-Warner SA, Orav J, Wu K, Fuchs CS, et al. Association of Physical Activity by Type and Intensity With Digestive System Cancer Risk. JAMA Oncol. 2016;2:1146-53. 